\title{
Erratum: Linking partial and quasi dynamical symmetries in rotational nuclei [Phys. Rev. C 89, 041302(R) (2014)]
}

\author{
C. Kremer, J. Beller, A. Leviatan, N. Pietralla, G. Rainovski, R. Trippel, and P. Van Isacker \\ (Received 14 September 2015; published 29 September 2015)
}

DOI: 10.1103/PhysRevC.92.039902

PACS number(s): 21.60.Fw, 21.10.Re, 21.60.Ev, 27.70.+q, 99.10.Cd

We used an erroneous structure parameter $\chi=-0.53$ in our Table I for the nucleus ${ }^{162} \mathrm{Gd}$. The correct structure parameter is $\chi=-0.30$ as given in Ref. [1]. This changes the fourth data row of our original table to:

TABLE I. Corrected values of the data row for ${ }^{162} \mathrm{Gd}$ in Table I of the original paper, as a result of using the correct structure parameter $\chi=-0.30$.

\begin{tabular}{lccccccccc}
\hline \hline Nucleus & $N$ & $\xi$ & $\chi$ & $\Delta \sigma_{0}$ & $f_{\sigma=\mathrm{N}}^{(0)}$ & $\Delta \sigma_{2}$ & $f_{\sigma=\mathrm{N}}^{(2)}$ & $\Delta \sigma_{4}$ & $f_{\sigma=\mathrm{N}}^{(4)}$ \\
\hline${ }^{162} \mathrm{Gd}$ & 15 & 0.98 & -0.30 & 0.17 & $99.3 \%$ & 0.17 & $99.3 \%$ & 0.16 & $99.3 \%$ \\
\hline \hline
\end{tabular}

This change does not effect any results or conclusions of the original paper. The nucleus ${ }^{162} \mathrm{Gd}$ is closer to the valley of low $\sigma$ fluctuations than originally proposed.

[1] E. A. McCutchan, N. V. Zamfir, and R. F. Casten, Phys. Rev. C 69, 064306 (2004). 